\title{
Distinct transcriptional control and action of fibroblast growth factor receptor 4 in differentiating skeletal muscle cells
}

\author{
ShunJiang $\mathrm{Yu}^{1}$, Lei Zheng ${ }^{1}$, Denny K Trinh ${ }^{1}$, Sylvia L Asa ${ }^{2}$ and Shereen Ezzat ${ }^{1}$ \\ ${ }^{1}$ Department of Medicine, Mount Sinai Hospital and University of Toronto, The Freeman Centre for \\ Endocrine Oncology and The Ontario Cancer Institute and ${ }^{2}$ Department of Pathology University Health \\ Network and University of Toronto, The Freeman Centre for Endocrine Oncology and The Ontario Cancer \\ Institute, 610 University Avenue, Toronto, Ontario, Canada M5G 2M9
}

\begin{abstract}
Although FGF signaling promotes myoblast proliferation and represses myogenic differentiation, one of the FGF receptors (FGFR), FGFR4, is expressed mainly in mature skeletal muscle. Disruption of FGFR4 signaling interrupts chick limb muscle formation. To determine the developmental regulation of FGFR4 expression, we compared the transcriptional control and action of FGFR4 in myoblasts and myotubes. We identified higher FGFR4 expression in differentiated myotubes than precursor myoblasts. FGFR4 promoter activity was localized within a region $115 \mathrm{bp}$ upstream of the transcription start site. Overlapping fragments of this promoter displayed a distinct difference when compared by electromobility shift assay (EMSA) using nuclear extracts from myoblasts and myotubes. While fragments $B(-95 /-56)$ and $C(-65 /-26)$ formed specific complexes in both cell types, these complexes were consistently more intense in myotubes than myoblasts. These complexes were efficiently competed by an Sp-type oligonucleotide and were supershifted by Sp1 and by Sp3 antibodies. Deletions of the Sp-binding sites in fragment B $(-95 /-56)$ confirmed their critical contribution to promoter activity. Moreover, Sp1 expression correlated with FGFR4-expression in myotubes. To determine whether FGFR4 expression regulates myoblast differentiation, we infected a soluble dominant-negative FGFR4containing adenovirus into these cells. This significantly impeded Erk1/2 phosphorylation and differentiation of myoblasts into MHC-expressing myotubes. Our findings point to distinct transcriptional regulation and action for FGFR4 in differentiating skeletal muscle cells.
\end{abstract}

Laboratory Investigation (2004) 84, 1571-1580, advance online publication, 4 October 2004; doi:10.1038/labinvest.3700187

Keywords: fibroblast growth factor receptor 4; FGF; myoblasts; Sp1; Sp3

Myogenic cells proliferate as mononucleated myoblasts before ultimately differentiating into multinucleated skeletal muscle fibers. This process is governed by signaling cascades orchestrated by a number of growth factors. Of these, members of the fibroblast growth factor (FGF) and FGF receptor (FGFR) family have been implicated in sustaining myoblast proliferation and possibly delaying their differentiation. ${ }^{1}$

The FGF family is composed of 22 members (FGFs 1-14 and 16-23), each possessing a conserved 120-amino-acid core domain. These ligands are organized into six groups based on phylogenetic

Correspondence: Dr S Ezzat, MD, University of Toronto-Mt. Sinai Hospital, 600 University Ave. \#437, Toronto, Ontario, Canada M5G-1X5.

E-mail: sezzat@mtsinai.on.ca

Received 15 July 2004; revised 19 August 2004; accepted 24

August 2004; published online 4 October 2004 relationships. FGFs bind with low affinity to heparan sulfate proteoglycans (HSPGs) present on most cell surfaces and extracellular matrices. FGF signaling is mediated through four distinct FGF receptors (FGFR1-4) that form a complex family of transmembrane receptor tyrosine kinases (RTKs). ${ }^{2}$ Each prototypic receptor is composed of three immunoglobulin (Ig)-like extracellular domains, two of which are involved in ligand binding, a single transmembrane domain, a split tyrosine kinase, and a $\mathrm{COOH}$-terminal tail with multiple autophosphorylation sites. ${ }^{2}$ Multiple forms of cellbound or secreted forms of FGFR1, 2 and 3 are generated by alternative transcription, alternative initiation, alternative splicing, exon switching, or variable polyadenylation. ${ }^{3,4}$ Alternative splicing results in a secretable first Ig-like domain and a separate membrane-bound 2 Ig form. ${ }^{5}$ Polyadenylation downstream of exons 3,6 or 7 results in termination of translation, yielding secreted 
receptors only. Soluble forms of the extracellular domain of FGFR1 may bind FGFs in blood. ${ }^{6}$ In FGFRs 1, 2 and 3, alternative RNA splicing of one of two exons results in two alternate forms of the second half of the third Ig-like domain; exon 8 encodes the IIIb form or exon 9 encodes the IIIC form. Whereas FGFR1, 2 and 3 are known to have multiple isoforms, FGFR4 until relatively recently was believed to have none. ${ }^{7-9}$

Many FGFs display high-affinity interactions with multiple FGFRs, while some activate unique receptors or receptor isoforms. Most FGFs have demonstrated mitogenic activity in a variety of systems. However, a few, including FGFs 11-14, 19, and 21-23, do not display proliferative functions. ${ }^{10}$ The mitogenic capability of an FGF is likely a function of the FGFR(s) with which it interacts. For example, FGF-19 plays a role in regulating cholesterol and bile acid synthesis through a unique non-heparindependent interaction with FGFR4. ${ }^{11}$ An essential role for FGF23 in physiologic regulation of phosphate and vitamin D metabolism has also emerged. ${ }^{12}$

FGF-1 and FGF-2 possess well-documented mitogenic activity on skeletal muscle cells and both can activate FGFR1 in proliferating myoblasts. ${ }^{13}$ FGF-2 stimulation of myoblasts induces SHP-2 complex formation with FRS2 and induces Erk activity and Elk-1 transactivation. Conversely, overexpression of SHP-2 potentiates the suppressive effects of FGF-2 on muscle-specific gene expression and myogenesis $^{14}$ further highlighting the potential importance of FGFR signaling in myogenic development.

Recent evidence, however, has challenged the general view that FGF signaling can delay myogenic differentiation. Loss of FGFR4 signaling was shown to result in impaired skeletal muscle development in chick embryos. ${ }^{15}$ FGFR4 was found to be overexpressed in tumors arising from skeletal muscle. ${ }^{16}$ Little is known, however, about FGFR4 regulation in general or in differentiating skeletal muscle cells.

In this report, we sought to obtain insight into the regulatory mechanisms governing FGFR4 gene regulation in myoblasts compared with myotubes. We mapped the elements required for promoter activity and identified relevant transcription factors in the extremes of cell differentiation to identify potential developmental mechanisms for FGFR4 regulation and function.

\section{Materials and methods}

\section{Cell Culture}

The mouse C2C12 skeletal muscle cell line was cultured in DMEM containing $10 \%$ fetal bovine serum (GIBCO BRL), $2 \mathrm{mM}$ L-glutamine and $100 \mathrm{IU} /$ $\mathrm{ml}$ penicillin and $100 \mu \mathrm{g} / \mathrm{ml}$ of streptomycin at $37^{\circ} \mathrm{C}$ in a $5 \% \mathrm{CO}_{2}$ humidified incubator. These cells initially have a myoblast phenotype with absence of muscle markers including myosin heavy chain (MHC) expression. As myoblasts reached 90\% confluence, the medium was changed to DMEM containing 5\% horse serum (GIBCO BRL). Four to 6 days of additional incubation were required for myotube differentiation. This stage was identified by classical tube formation and expression of MHC.

\section{Western Blotting Analysis}

Protein concentrations were determined by the BioRad protein assay. Equal amounts of protein $(50 \mu \mathrm{g})$ from whole cell lysates or nuclear fractions were solubilized in $2 \times$ SDS-sample buffer, separated on SDS-8\% polyacrylamide gels and transferred to nitrocellulose. Blots were incubated with rabbit polyclonal anti-FGFR4, Sp1, or Sp3 antibodies all from Santa Cruz at a concentration of 1:1000, or $\beta$-actin (Sigma) at 1:500. MHC expression was detected using anti-MF20 antibody (kindly provided by M Rudnicki, Ottawa). Total and phosphorylated forms of the Erk1/2 forms of MAPK were detected using total and phospho-specific antibodies respectively (1:1000; New England Biolabs).

\section{Plasmids}

Promoter analysis of the FGFR4 gene was performed with the assistance of gene finder (http:// genome.cbs.dtu.dk/htbin/nph-webface). Possible transcription start sites were detected with TRANSFACPromoter 2.0 Prediction Program. To generate the $-1133 /+99$ fragment, PAC\# 32C5 was used for PCR using an upstream primer containing a KpnI restriction site, and a downstream primer containing a BgIII or HindIII site, permitting subcloning into the corresponding sites of the multiple cloning site of the promoterless firefly luciferase expression vector pGL3 (Promega, WI, USA) to produce a $\mathrm{P}$ $(-1133 /+99)$-Luc construct. Other reporters $\mathrm{P}(-855 /+99)$-Luc, $\mathrm{P}(-173 /+99)-$ Luc and, $\mathrm{P}(-115 /$ $+99)-L u c$ were generated by restriction of the $\mathrm{P}(-1133 /+99)-L u c$ construct with StuI, XhoI, and SmaI, respectively. P $(-535 /+99)-L u c$ was constructed by PCR using a primer containing an FspI restriction site. The $\mathrm{P}(+13 /+99)$-Luc construct was synthesized (Sigma, Ontario, Canada) and similarly positioned into the pGL3 basic vector. The construct $\mathrm{P}(-1133 /-173)$-Luc was generated by deleting the $3^{\prime}$ end of the $(-1133 /+99)$-Luc construct using $X h o I$ with subsequent ligation into the corresponding KpnI and XhoI sites in pGL3. The orientation and sequences of all constructs were verified by restriction analysis and nucleotide sequencing.

The pPAC expression vectors encoding Sp1 and Sp3 were kindly provided by Dr P Marsden (University of Toronto) and were cloned into the mammalian pcDNA3.1 (InVitrogen) vector. Sequence fidelity was confirmed by direct sequencing and protein expression monitored by Western 
immunoblotting. An FGFR4 expression vector was used as previously described. ${ }^{17}$

To abrogate FGFR4 signaling, we used a soluble dominant-negative $(\mathrm{dn})$ strategy. In addition to deletion of the cytoplasmic domain, the FGFR transmembrane domain is substituted with the human Ig heavy chain hinge and IgG1 Fc domains thus creating a stable and secretable chimeric protein dnFGFR-HFc. This dnFGFR efficiently interferes with membrane-anchored FGFR signaling ${ }^{18}$ and abrogates FGF1-induced MAPK (Erk1/2) stimulation transduced by FGFR4 without affecting endogenous FGFR1-4 expression. ${ }^{7}$ To maintain stable expression in differentiating cells, we adopted an adenoviral approach for gene transfer. ${ }^{19}$ The dnFGFR-HFc cDNA was subcloned into pACCMV-pLpA vector and cotransformed with pjM17 plasmid into HEK 293 cells. Successful recombination between the two plasmids results in recombinant viruses encoding dnFGFR-HFc inserts and the control Ad- $\beta$ gal. Following plaque formation, viruses were amplified in HEK 293 cells and purified. Plaque-forming units (PFU) were quantified using a commercial Adeno-X rapid titer kit (Boheringer). Chimeric dnFGFR-HFc protein expression was monitored by immunoblotting with antiHFc antibody at 1:2000 (Dako).

\section{DNA Deletion and Mutagenesis}

The $5^{\prime} \mathrm{Sp} 1$ binding site in fragment B of the FGFR4 promoter (Figure 3a) was deleted by ApaI digestion. This corresponds to FGFR4 genomic position 1055 and generated the reporter construct $\mathrm{P}(-86 /+99)$ Luc. The remaining three Sp1 binding sites were deleted using mutagenic PCR primers. For deletion of the second binding site the mutagenic sense primer used was 5'-CCGAGCAGGAGGGTTCGGGC CCGAGGG-3' (corresponding to FGFR4 genomic positions 1059-1076). For the third Sp1 site the mutagenic sense primer used was 5'-GCGGGC CCAGGTTCGGGGCGGGACAGGAG-3' (corresponding to FGFR4 genomic positions 1073-1102). For targeting the fourth Sp1 site the mutagenic sense primer used was 5'-GCCCGAGGTTCGGTTCGGGA CAGGAGGTG-3' (corresponding to FGFR4 genomic positions 1077-1105). The common antisense primer was 5'-GCTCCTCCGCCGCCTCACTC-3'. The generated reporter constructs were $\mathrm{P}(-80 /+99)$ Luc, $\mathrm{P}(-66 /+99)-\mathrm{Luc}$, and $\mathrm{P}(-62 /+99)$-Luc, respectively. The PCR conditions used were as follows: denaturation at $95^{\circ} \mathrm{C}$ for $3 \mathrm{~min}$, followed by 30 cycles of $95^{\circ} \mathrm{C}$ for $40 \mathrm{~s}, 56^{\circ} \mathrm{C}$ for $45 \mathrm{~s}$, and $72^{\circ} \mathrm{C}$ for $1 \mathrm{~min}$, and finally $72^{\circ} \mathrm{C}$ for $7 \mathrm{~min}$. All inserts with deletion and/or mutation were confirmed by restriction digestion and nucleotide sequencing.

\section{Transfection and Luciferase Assays}

Plasmid reporters were prepared by column chromatography (Qiagen, Mississauga, ON, Canada) for sequencing and transfections. Cells were transfected by the Lipofectamine reagent (Invitrogen) according to the manufacturer's protocol. Cells were plated into 12 wells at a density of $3-5 \times 10^{5}$ cells and transfected the following day after plating with $3 \mu \mathrm{l} /$ well of lipofectamine and $0.5-2 \mu \mathrm{g}$ of DNA per well as indicated. The total amount of transfected DNA was kept constant by adding empty vector. Transfection efficiency was monitored by simultaneous co-transfection with a $\beta$-galactosidase control expression plasmid CMV- $\beta$ gal $(20 \mathrm{ng} /$ well). At $24 \mathrm{~h}$ following transfection, cells were lysed in buffer containing $25 \mathrm{mM}$ glycylglycine, $15 \mathrm{mM} \mathrm{MgSO}_{4}$, $4 \mathrm{mM}$ EGTA, $1 \%$ Triton X, and $1 \mathrm{mM}$ DTT. Luciferase activity was measured for $20 \mathrm{~s}$ in a Lumat LB 9507 luminometer. $\beta$-Galactosidase activity was measured to normalize for variations in transfection efficiency. Promoter activity of each construct was expressed as firefly luciferase/ $\beta$-gal activity. Each experiment was independently performed on three separate occasions with triplicate wells in each group.

\section{Preparation of Nuclear Extracts}

Nuclear extracts from myoblasts and myotubes $(24 \mathrm{~h}$ of culture after plating for myoblasts and 4-7 days for myotubes) were prepared by washing cells in $1 \times$ PBS cold and lysed in $100 \mu \mathrm{l}$ of buffer containing (10 mM HEPES pH 7.9, $1 \mathrm{mM}$ DTT, $1 \mathrm{mM}$ EDTA, $60 \mathrm{mM} \mathrm{KCl}, 0.5 \% \mathrm{NP}-40,1 \mathrm{mM}$ PMSF) $5 \mathrm{~min}$ on ice. The pellet was resuspended into $100 \mu \mathrm{l}$ of the nuclear resuspension buffer $(0.25 \mathrm{mM}$ Tris- $\mathrm{HCl} \mathrm{pH}$ 7.8, $60 \mathrm{mM} \mathrm{KCl,} 1 \mathrm{mM}$ DTT, $1.5 \mathrm{mM} \mathrm{PMSF)} \mathrm{and}$ lysed with three cycles of freezing and thawing to $37^{\circ} \mathrm{C}$. After centrifugation at $13000 \mathrm{rpm}$ for $10 \mathrm{~min}$ at $4^{\circ} \mathrm{C}$, the clear supernatant was collected and stored at $-80^{\circ} \mathrm{C}$ for further analysis. Protein concentrations were determined by the Bio-Rad protein assay.

\section{Electrophoretic Mobility Shift Assays (EMSA)}

Oligonucleotides were end-labeled with ${ }^{32} \mathrm{PdATP}$ using the T4 DNA kinase. Overlapping doublestranded oligonucleotide fragments of FGFR4 (Figure 3a) between $-115 /+99$ were used as probes and for competition in EMSAs as follows: fragment B ( -95 to -56$), 5^{\prime}$-GAAGGAGGGGCGGGCCCGAGCA GGAGGGGGCGGGCCCGAG-3', fragment C ( -65 to -26), sense: $5^{\prime}$-CGGGCCCGAGGGGCGGGGCGGGACAGGAGGTGGGCCGATC- ${ }^{\prime}$, fragment D $(-35$ to $+4), 5^{\prime}$-TGGGCCGCTCGCGGCACGCCGCCGTCGCG GGTACATTCCT-3'; fragment E $(-5$ to +24$)$, $5^{\prime}$ GTACATTCCTCGCTCCCGGCCGAGGAGCGC-3'; fragment $\mathrm{F}(+15$ to +54$), 5^{\prime}$-CGAGGAGCGCTCGGG CTGTCTGCGGACCCTGCCGCGTGCA-3'; and fragment $\mathrm{G}(+49$ to +99$), 5^{\prime}$-CGTGCAGGGGTCGCGGC CGGCTGGAGCTGGGAGTGAGGCGGCGGAGGAGC$3^{\prime}$. Fragment A contains long stretches of $G^{\prime}$ s $(-115$ 
to -86): $5^{\prime}$-GGGTGGGGGGGGGGGGCGTGGAAGGA

GGGG-3' could not be synthesized.

Competitor oligonucleotides containing transcription binding sites were as follows: Sp1: 5'-ATTCGATCGGGGCGGGGCGAGC-3'， Ets-1: 5'-GGGCTGC TTGAGGAAGTATAAGAAT-3'; Ikaros: 5'-AAGAA GCGGGAGTGACAGG-3' and their complementary strands were synthesized by Sigma (Sigma, Oakville, ON, Canada). The complementary strands were annealed in a buffer of $10 \mathrm{mM}$ Tris-Cl pH 8.0, $50 \mathrm{mM} \mathrm{NaCl}$, and $1 \mathrm{mM}$ EDTA. Gel-shift probes were radiolabeled using T4 DNA kinase (Invitrogen), and purified with G50 spin column. For EMSA, $100 \mathrm{cpm}$ of labeled probe was incubated with $5 \mu \mathrm{g}$ nuclear extracts at room temperature for $30 \mathrm{~min}$ in a binding reaction consisting of $20 \mathrm{mM}$ HEPES ( $\mathrm{pH}$ 7.9), $50 \mathrm{mM} \mathrm{KCl}, 1 \mathrm{mM}$ EDTA, $1 \mathrm{mM}$ DTT, $0.5 \mathrm{mM} \mathrm{MgCl}{ }_{2}$, $2 \%$ glycerol, and $0.5 \mu \mathrm{g}$ poly(dI-dC) (Pharmacia, NJ, USA) in a final volume of $20 \mu \mathrm{l}$. For competition assay, molar excess of unlabeled oligonucleotide or antibody to the relevant transcription factor was added $30 \mathrm{~min}$ before addition of radiolabeled probe. Samples were electrophoresed on $4 \%$ polyacrylamide nondenatured gels containing $0.5 \%$ Tris-borate buffer and $2 \%$ glycerol. Gels were dried under vacuum and autoradiographed.

\section{Statistical Analysis}

Data are presented as mean \pm s.d. Differences were assessed by Student's paired $t$-test. Significance level was assigned at $P<0.05$.

\section{Results}

\section{Characterization of FGFR4 Expression in Myoblasts and Myotubes}

To compare the expression of FGFR4 in myocytes of different degrees of differentiation, we examined myoblasts and myotubes by Western immunoblotting. Using an antibody that specifically recognizes FGFR4, myotubes revealed higher levels of FGFR4 reactivity than myoblasts (Figure 1). The mature glycosylated $110 \mathrm{kDa}$ FGFR4-reactive band comigrated with lysates from HEK 293 cells transfected with FGFR4. ${ }^{8}$ Further, this band was abolished when lysates were preincubated with FGFR4 blocking peptide (Figure 1). The stage of myocyte differentiation was confirmed by the detection of MHC expression in myotubes but not in myoblasts (Figure 1).

\section{Characterization of the FGFR4 Promoter in Myocytes}

Interactive elements, such as TATA or CAAT boxes, often regulate the assembly and efficiency of the basic transcriptional machinery. As with other FGFRs, the FGFR4 gene lacks a classic TATA box but includes a $5^{\prime}$ upstream region that is rich in GC

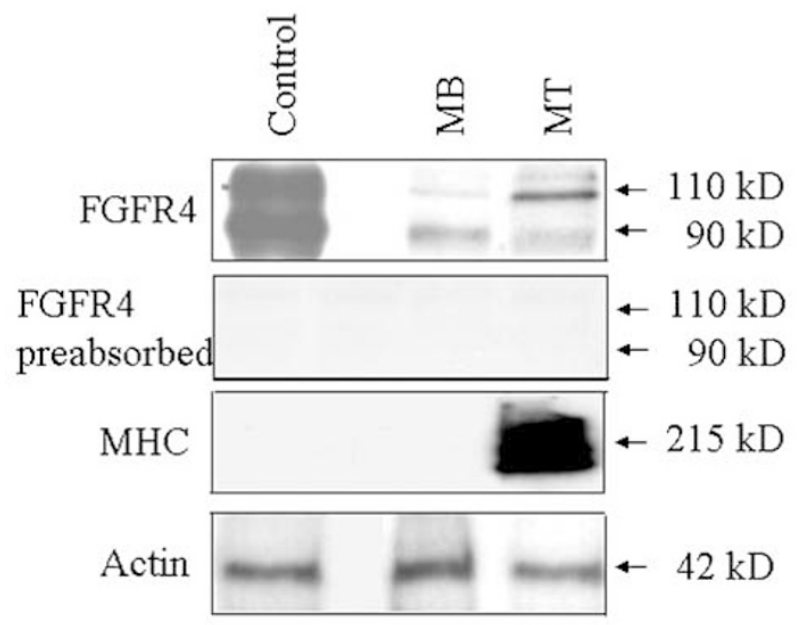

Figure 1 FGFR4 expression in skeletal myoblasts vs myotubes. Whole cell lysates from C2C12 cells at the myoblast (MB) or myotube (MT) phase of differentiation were subjected to immunoblotting using antibodies to FGFR4, myosin heavy chain (MHC; antibody MF-20) or actin as indicated. The control lane represents HEK 293 cells transfected with FGFR4 and is followed by an empty lane. FGFR4 expression is prominent in myotubes and preabsorption of the FGFR4 antiserum with peptide abolishes the $110 \mathrm{kDa}$ reactive band. MHC, a marker of myotube differentiation, confirms the differentiation of these cells.

residues. The latter contains many consensus motifs that would predict recruitment of RNA polymerase II and initiate gene transcription. We had previously isolated the $5^{\prime}$-upstream region of the FGFR4 gene from the PAC\#32C5. ${ }^{20}$ The region -1133 to +99 from the transcription start site $^{21,22}$ was placed upstream of the luciferase-reporter vector pGL3basic. A series of deletion analysis was used to define the minimal promoter and fragments essential for functional activity. This activity was situated within the region of $\mathrm{P}(-115 /+99)$ (Figure 2) in myoblasts. Serial $5^{\prime}$-deletions of the FGFR4 promoter region to $+13 /+99$ markedly abrogated promoter activity while the $3^{\prime}$-deleted construct $\mathrm{P}(-1133$ / -173 ) revealed no significant promoter activity.

\section{FGFR4 Minimal Promoter Relies on Multiple Sp1-Binding Sites}

To determine which factors are most relevant in mediating FGFR4 promoter activity in myoblasts compared to myotubes, we screened potential candidates using EMSA. EMSAs were performed using nuclear extracts from myoblasts and myotubes with overlapping $40-50 \mathrm{bp}$ oligonucleotide fragments derived from the $214 \mathrm{bp}$ minimal FGFR4 promoter (Figure 3a). DNA-protein complexes were detected when nuclear extracts from myotubes and myoblasts were allowed to interact with fragments B $(-95 /-56)$ and $C(-65 /-26)$. These complexes were consistently more intense with myotube than with myoblast nuclear extracts (Figure $3 \mathrm{~b}$ ). Based on 


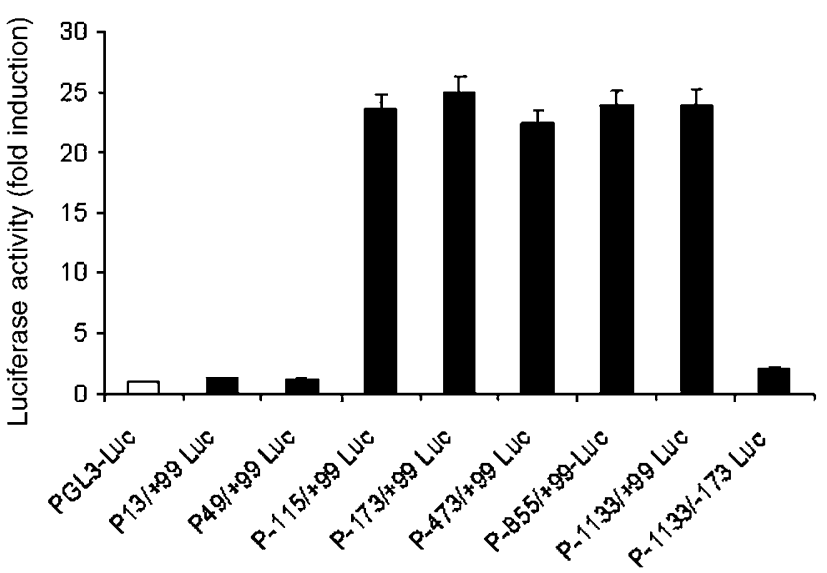

Figure 2 Functional characterization of the FGFR4 promoter in differentiating skeletal muscle cells. The effects of sequences spanning the proximal $5^{\prime}$ region of FGFR4 from -1133 to +99 on luciferase reporter activity were assessed in transiently transfected myoblasts as indicated. A series of deleted promoter fragments were generated by PCR as described in Materials and methods, ligated and subcloned upstream of the promoterless luciferase reporter gene pGL3-basic vector as indicated. Note that serial $5^{\prime}$-deletions of the FGFR4 promoter region to $\mathrm{P}(+13 /+99)$ Luc markedly abrogates luciferase activity, and that the $3^{\prime}$ - deleted construct $\mathrm{P}(-1133 /-173)$-Luc shows minimal activity. Data are presented as the mean luciferase activity adjusted for $\beta$-gal activity ( + s.d.) and compared with control wells from three independent experiments $(P<0.005)$.

sequence prediction, fragments $\mathrm{B}$ and $\mathrm{C}$ each contain 2 Sp-binding sites (Figure 3a). Consistent with this prediction, these complexes were competed by an Sp-type oligonucleotide (Figure 3c). Moreover, the upper (slower) migrating band of this complex was supershifted by an Sp1 antibody while the lower (faster) migrating component of the complex was supershifted by Sp3 antibody (Figure 3c).

In contrast to these Sp findings, we found no evidence for significant binding by other predicted factors including Ap1, Ap2, Ap4, CREB, Yy-1, Ets, and Ikaros-type binding sites ${ }^{9,20,23}$ in either myoblasts or myotubes (data not shown).

\section{Effects of Sp1 and Sp3 on the Regulation of FGFR4 in Myocytes}

To determine the functional contribution of each of the four Sp-binding sites in fragments B and C in the FGFR4 promoter, we individually deleted each of these sites. Deletion of the most $5^{\prime}$ site in fragment B resulted in the most pronounced loss of promoter activity (Figure 4a). Moreover, loss of this $5^{\prime}-\mathrm{Sp}$ binding site resulted in near complete abrogation of responsiveness to Sp1 cotransfection (Figure 4b). In contrast to the Sp1 effects, Sp3 transfection had minimal effect on FGFR4 promoter activity (Figure 4b). Similarly, Ets1 transfection had no appreciable effect on FGFR4 promoter activity in myoblasts or myotubes (data not shown).
To examine whether FGFR4 expression correlates with Sp1 expression in differentiating myocytes, we compared the protein expression of Sp1 by Western blotting in myoblasts with myotubes. Nuclear fractions from myotubes revealed higher levels than myoblasts of the $105 \mathrm{kDa}$ Sp1 isoform with no difference in levels of the smaller $95 \mathrm{kDa}$ isoform. No appreciable difference in Sp3 expression ( 130 and $76 \mathrm{kDa}$ ) between the two phases of myocyte differentiation was detected (Figure 4c).

\section{Adenoviral-Mediated Transduction of Dominant-Negative FGFR Impedes Myocyte Erk1/2 Activation and Cellular Differentiation}

To determine the functional role of FGFR4 on myocytes, we examined the effect of dominantnegative FGFR4 transduction on progression to myotube differentiation. Myoblasts were infected with an adenovirus expressing a soluble dominantnegative FGFR (dnFGFR-HFc) or the empty-vector adenovirus. Western immunoblotting confirmed the diminished expression of the differentiation marker MHC by cells infected with dnFGFR as well as interruption of Erk1/2 phosphorylation (Figure 5a). Cells were examined daily and photographed using phase-contrast microscopy after 4 days of infection. As seen in Figure 5b, more than $80 \%$ of controlinfected myoblasts differentiate into large myotubes. In contrast, a dose-dependent inhibition of myotube formation was evident in cells transduced with dnFGFR-HFc.

\section{Discussion}

We have compared the expression and promoter activity of FGFR4 in myocytes of different degrees of differentiation. We examined the cis-regulatory elements in the proximal region of the FGFR4 promoter that render positive transcriptional activity in high FGFR4-expressing myotubes with those from weak-FGFR4-expressing myoblasts. We demonstrate that multiple binding elements mainly in fragments $B(-95 /-56)$ and $C(-65 /-26)$ of the FGFR4 promoter contain Sp binding sites. Our findings point to the importance of Sp1 binding sites in the region of fragment $B$ as particularly important for FGFR4 expression in differentiating myotubes. Further, we demonstrate that dominantnegative interruption of FGFR4 impedes myotube formation in $\mathrm{C} 2 \mathrm{C} 12$ cells. These findings begin to address the mechanisms underlying the differences in FGFR4 expression and its role in differentiating myocytes.

Sp1 has been shown to play a significant role in the regulation of other FGFRs. For example, analysis of the mouse FGFR1 gene reveals the presence of consensus sequences for binding sites of the transcription factors Sp1 and the absence of TATA and CAAT sequence motifs. ${ }^{24}$ Transfection 
1576

of 5 - regulatory region into NIH 3 T3 cells defined a minimal promoter within the region defined by -106 and +104 of FGFR1. ${ }^{24}$ Similarly, deletion

a

Fragment $B(-95$ to -56$)$

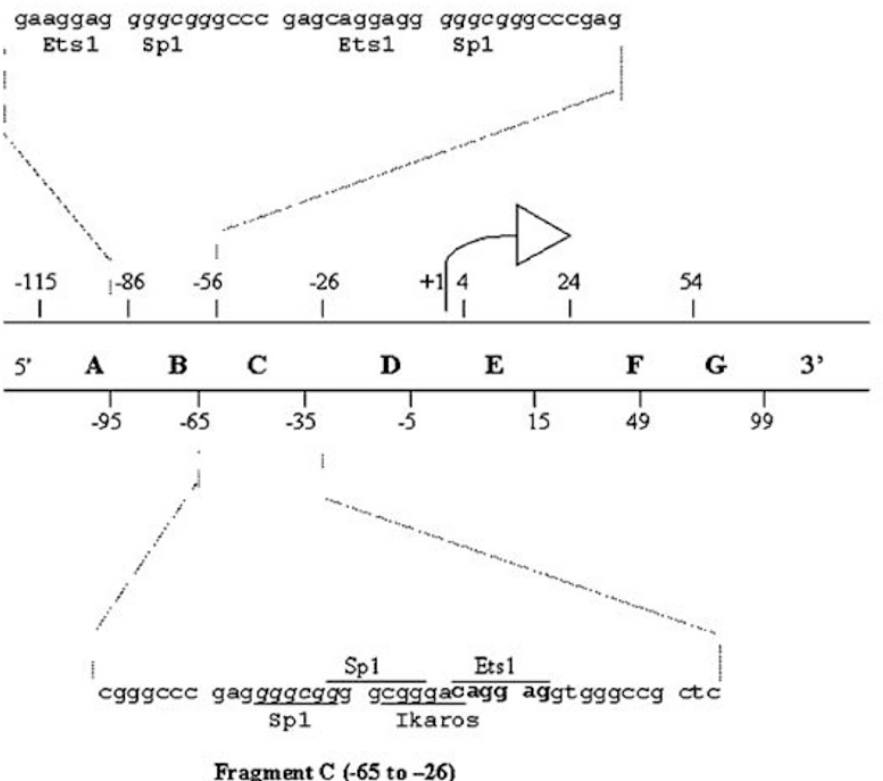

C

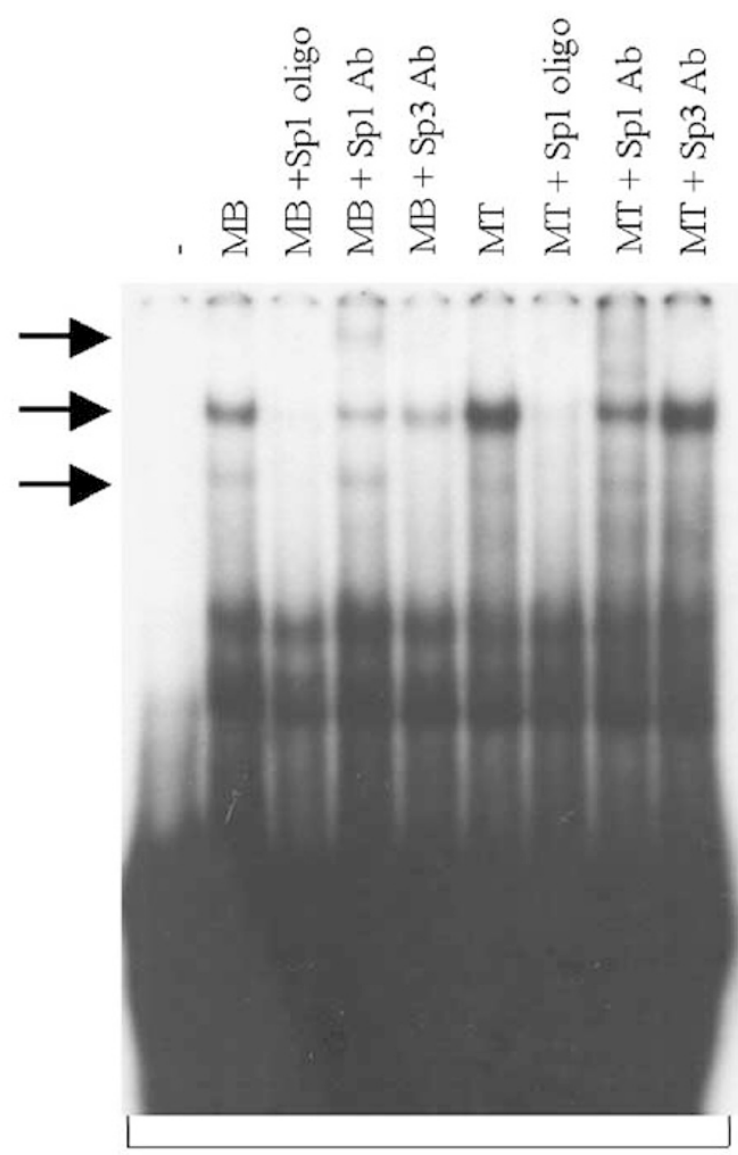

B analysis of the avian FGFR1 promoter reveals a $78 \mathrm{bp}$ region containing multiple $\mathrm{Sp} 1$ binding sites that confer a high level of FGFR1 promoter activity

b

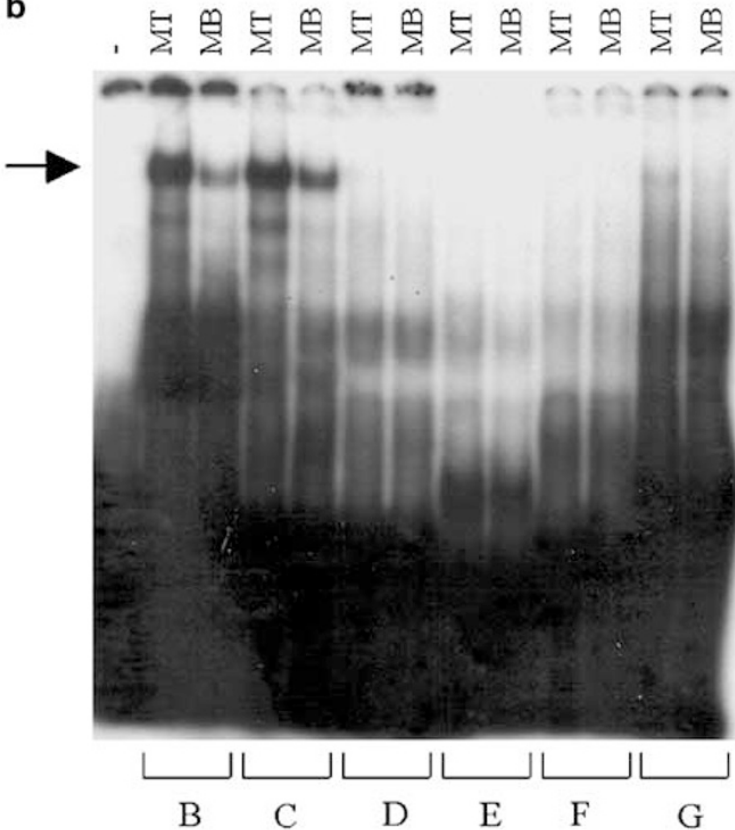

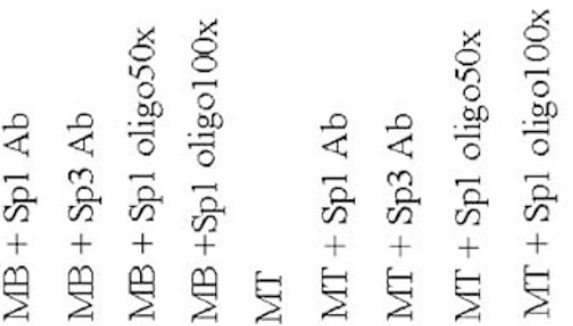


a

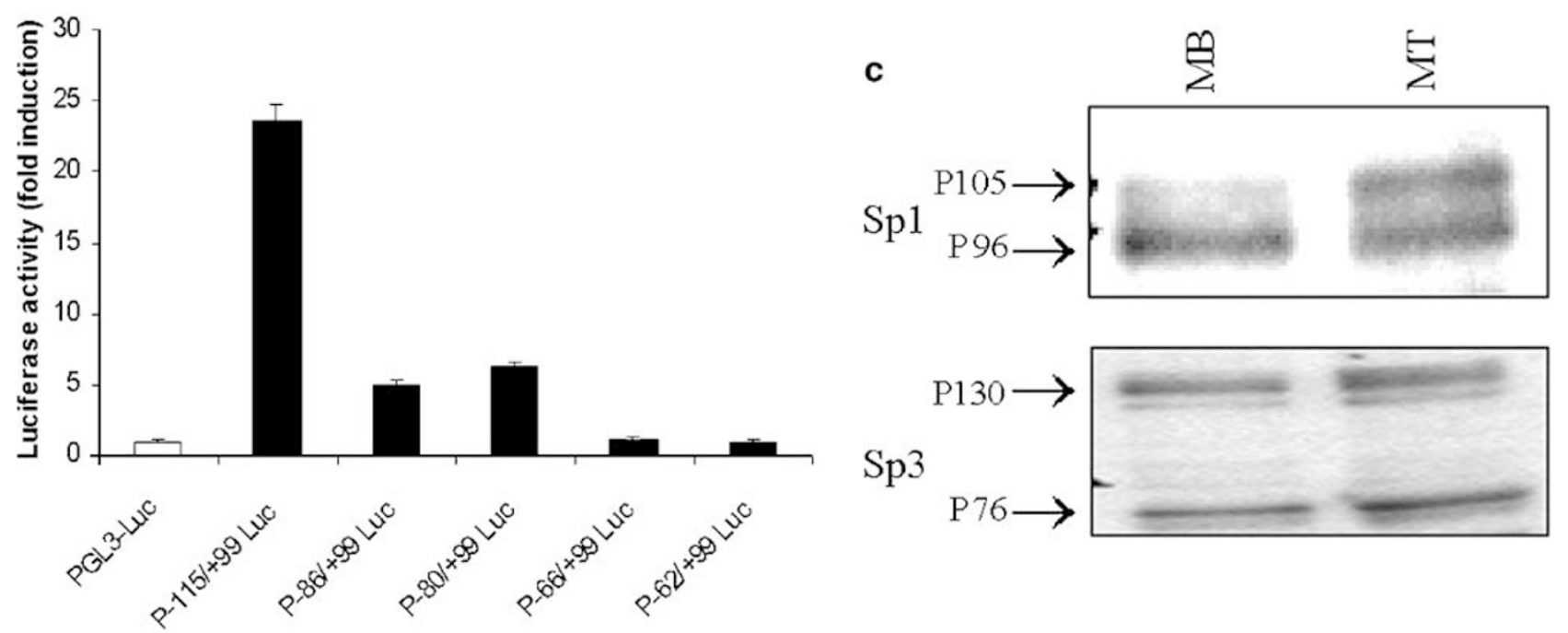

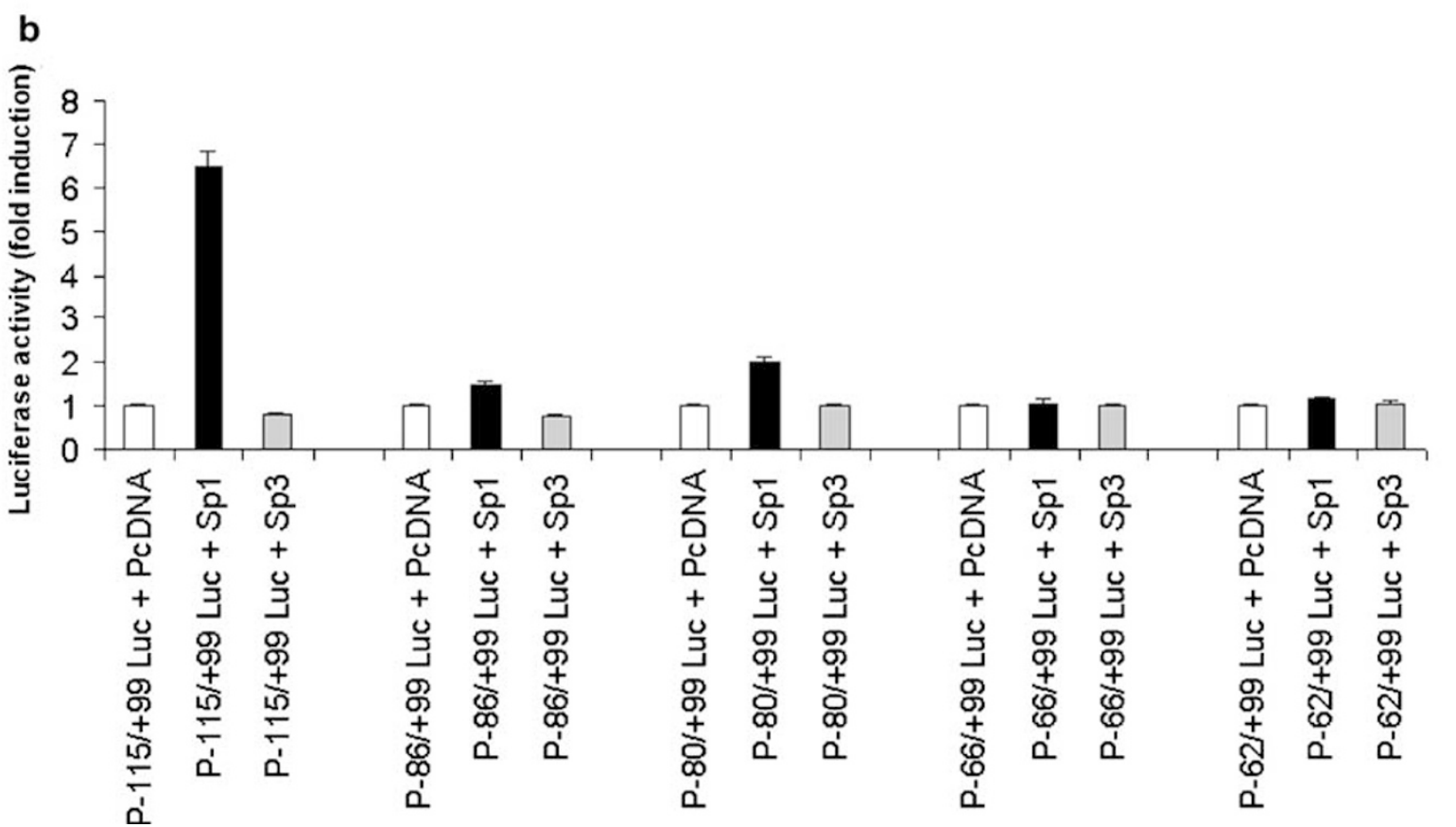

Figure 4 Sp1 but not Sp3 regulates FGFR4 transcriptional activity in skeletal muscle cells. (a) Myoblasts were transiently cotransfected with the wild-type minimal FGFR4 $\mathrm{P}(-115 /+99)$-Luc promoter or progressive Sp-binding deletions in fragments B $(-86 /+99)(-80 /$ $+99)$ and $C(-66 /+99)(-62 /+99)$ as indicated. Note the marked loss of promoter activity with deletion of the Sp-binding sites in fragment B. (b) Myoblasts were transiently cotransfected with the progressive Sp-deleted minimal FGFR4 promoter and pcDNA3.1encoding Sp1 or Sp3 as indicated. Note efficient activation of FGFR4 promoter activity by Sp1 an effect which was lost with deletion of the $5^{\prime}$ Sp-binding site in fragment B $(-86 /+99)$. Note ineffectiveness of Sp3 in measurably influencing promoter activity. All transfections included corresponding empty control vectors along with $20 \mathrm{ng}$ of pCMV $\beta$ gal to normalize for transfection efficiency. The results are mean + s.d. derived from three independent experiments with each construct performed in duplicate. (c) Extracts were compared with Western immunoblotting. Myotubes (MT) demonstrated higher Sp1 levels than myoblasts (MB). Sp3 reactivity was similar in both cell stages of differentiation and demonstrates comparable protein loading.

Figure 3 Characterization of transcription factor binding elements in the FGFR4 promoter. (a) The FGFR4 (-115/ + 99) minimal promoter was divided into overlapping fragments $(A-G)$ which were used to characterize individual binding factors in differentiating skeletal muscle cells. Note the presence of two distinct Sp-binding sites (italics) in each of fragments B and C and the flanking Ikaros and Ets-type binding sites in fragment C. (b) The overlapping fragments (A-G) were used to compare binding with nuclear extracts from the myoblasts (MB) and myotubes (MT). The (-) lane represents omission of nuclear protein. Only fragments B and C formed distinct DNA-protein complexes (arrow) that were more evident in myotubes compared with myoblasts. (c) Fragments B and C were further tested as probes to compare their binding interactions with nuclear extracts from myoblasts (MB) and myotubes (MT) as indicated. Fragments B and C formed consistently stronger complexes with MT where the slower migrating band (middle arrow) is supershifted (top arrow) by Sp1 antibody and the faster migrating band (bottom arrow) is abolished by Sp3 antibody. Both complexes are competed by 50- and 100-fold excess of Sp1 oligonucleotide as indicated. 

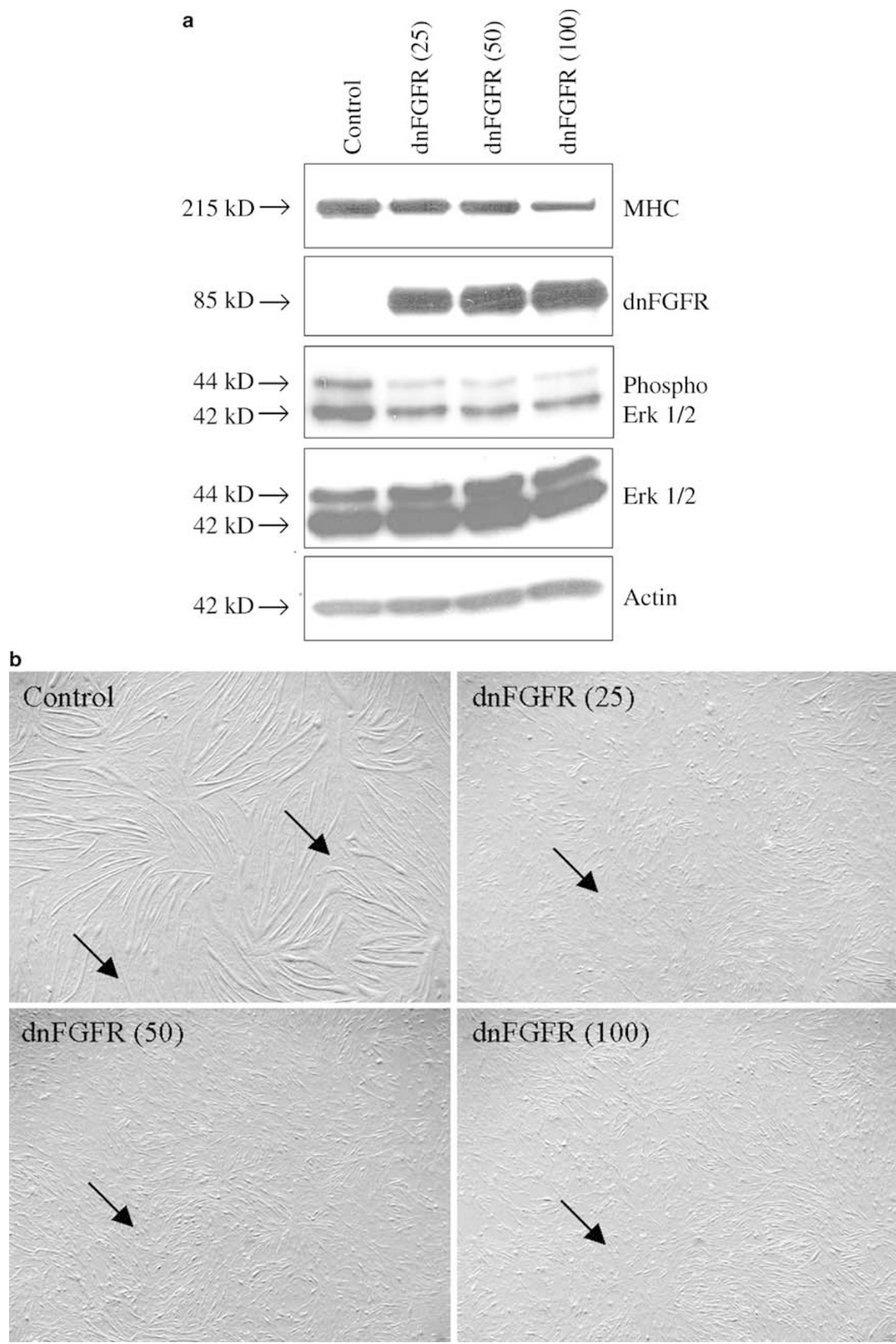

dnFGFR (100) 
in myoblasts. ${ }^{25}$ The FGFR2 promoter has been localized to a CpG island encompassing the $5^{\prime}$ end, ${ }^{26}$ lacking classical cis-regulatory motifs. Sequence analysis of the FGFR3 promoter also reveals multiple transcription binding sites including five classical Sp1 sites ${ }^{26}$ all situated within the first $200 \mathrm{bp}$ from the transcription start site. From these and our current studies, it may be concluded that for all FGFRs (FGFR1,2,3,4) as little as $100 \mathrm{bp}$ of sequence $5^{\prime}$ to the transcription initiation site confers significant transcriptional activity.

FGFR1 promoter studies have provided some insight into the possible mechanisms of FGFR1 regulation in skeletal muscle cells. The distal region of the FGFR1 promoter is located more than $1 \mathrm{~kb}$ upstream from the start of transcription and has been shown to positively regulate FGFR1 gene expression in myoblasts. ${ }^{25}$ This region contains two $\mathrm{Sp}$ transcription factor-binding sites, both of which are required for FGFR1 promoter activity in proliferating myoblasts. Although the distal Sp1 binding sites are required for full FGFR1 promoter activity and confer increased transcriptional activity, the distal region was not sufficient for promoter activity. Instead, the proximal region between -69 and -14 of the FGFR1 promoter appears to be more critical. However, in contrast to our findings with FGFR4 promoter, electromobility shift assays revealed that myoblast but not differentiated myotube nuclear proteins specifically bind to the ciselements in the FGFR1 promoter. ${ }^{27}$ There was no evidence of significant Sp3-mediated repression of the FGFR1 promoter. ${ }^{27}$ Similarly, we found no evidence for significant repression by Sp3 on FGFR4 regulation.

Our previous analysis of the FGFR4 promoter in pituitary cells identified a $214 \mathrm{bp}$ fragment containing 115 nucleotides upstream of the FGFR4 transcription start site as critical for promoter activity. ${ }^{20}$ Overlapping $40-50 \mathrm{bp}$ fragments of this minimal functional promoter were examined by EMSAs. Specific DNA-protein complexes were noted with two adjacent fragments. As shown here, these two fragments contain multiple $\mathrm{Sp}$ binding sites. In particular, fragment B contains two Sp sites that formed strong complexes with FGFR4-expressing myotubes in contrast with the low FGFR4-expressing myoblasts. Fragment C $(-65 /-25)$, however, also contains multiple predicted binding sites for Ap1, Ap2, Ap4, CREB, Yy-1, Ets, and Ikaros-type binding sites. ${ }^{23}$ The latter predicted binding element is flanked by two sites for Sp1. However, unlike lymphopoietic and pituitary cells, ${ }^{20}$ we found no evidence of Ikaros expression in either myoblasts or myotubes. Moreover, EMSA studies failed to identify significant binding for these other factors. Instead, our data emphasize the importance of Sp1 as an FGFR4 regulator in differentiated myocytes.

Thus far, the wealth of data has suggested a differentiation arrest mediated by FGFR signaling. Numerous studies have demonstrated that FGFs promote cell proliferation and repress terminal differentiation. ${ }^{28}$ In addition, genetic studies in Caenorhabditis elegans have demonstrated that FGF signaling plays an important role in myoblast migration. ${ }^{29}$ Thus it appears that FGFs have the capacity to promote cell proliferation, differentiation, and/or migration of myogenic precursors. FGFR1 transcription gradually decreases as muscle cell lines differentiate ${ }^{13}$ and this has been interpreted to indicate that FGFR1 plays an important role sustaining myoblast arrest along the myogenic lineage. We propose here that FGF action in myocyte differentiation needs to be reconsidered in the context of FGFR signaling contributions. While the weight of evidence has implicated FGFR1 in arresting myoblast differentiation, the contrasting pattern with FGFR4 expression and regulation shown here suggests distinct regulatory mechanisms for FGFR1 from FGFR4 in differentiating muscle cells. The dnFGFR approach we used here is not selective for FGFR4 alone and could potentially also interrupt FGFR1 expressed by these cells. However, given the arresting function of FGFR1, the dnFGFR-resulting blockade of myotube development shown here is consistent with a differentiating function for FGFR4. Taken together, our data are more in line with an emerging theme assigning FGFR4 a distinctly positive role in myogenesis and terminal skeletal muscle differentiation.

\section{Acknowledgements}

This work was supported by the Cancer Research Society and the Toronto Medical Laboratories.

\section{References}

1 Itoh N, Mima T, Mikawa T. Loss of fibroblast growth factor receptors is necessary for terminal differentiation of embryonic limb muscle. Development 1996;122:291-300.

2 Givol D, Yayon A. Complexity of FGF receptors: genetic basis for structural diversity and functional specificity. FASEB J 1992;6:3362-3369.

Figure 5 Dominant-negative FGFR transduction of myoblasts impedes myotube development. Myoblasts were infected with adenoviruses of varying amounts encoding a soluble dominant-negative FGFR or the empty control. (a) Western immunoblotting reveals diminished expression of the MHC differentiation marker by myoblasts (by just over 50\% as assessed by densitometry) 4 days following infection with dnFGFR in $\left(\times 10^{11} \mathrm{PFU}\right)$. Erk1/2 activation was also interrupted as determined by phospho-specific detection, but total levels were unchanged. The $\beta$-actin loading controls are shown below. (b) Myoblasts were photographed under phase-contrast microscopy 4 days after adenoviral infection as above. There is diminished myotube formation. The arrows point to small myoblasts that are all at the same size in these micrographs taken at the same magnification. 
3 Yan G, Wang F, Fukabori Y, et al. Expression and transformation of a variant of the heparin-binding fibroblast growth factor receptor $(\mathrm{flg})$ gene resulting from splicing of the exon at alternate $3^{\prime}$-acceptor site. Biochem Biophys Res Commun 1992;183:423-430.

4 Peters KG, Werner S, Chen G, et al. Two FGF receptor genes are differentially expressed in epithelial and mesenchymal tissues during limb formation and organogenesis in the mouse. Development 1992;114: 233-243.

5 Crumley G, Bellot F, Kaplow JM, et al. High-affinity binding and activation of a truncated FGF receptor by both aFGF and bFGF. Oncogene 1991;6:2255-2262.

6 Hanneken A, Ying W, Ling N, et al. Identification of soluble forms of the fibroblast growth factor receptor in blood. Proc Natl Acad Sci USA 1994;91:9170-9174.

7 Ezzat S, Zheng L, Yu S, et al. A soluble dominant negative fibroblast growth factor receptor 4 isoform in human mcf-7 breast cancer cells. Biochem Biophys Res Commun 2001;287:60-65.

8 Ezzat S, Zheng L, Zhu XF, et al. Targeted expression of a human pituitary tumor-derived isoform of FGF receptor-4 recapitulates pituitary tumorigenesis. J Clin Invest 2002;109:69-78.

$9 \mathrm{Yu}$ S, Asa SL, Weigel RJ, et al. Pituitary tumor AP2alpha recognizes a cryptic promoter in intron 4 of fibroblast growth factor receptor 4. J Biol Chem 2003;278:19597-19602.

$10 \mathrm{Fu} \mathrm{L}$, John LM, Adams SH, et al. Fibroblast growth factor 19 increases metabolic rate and reverses dietary and leptin-deficient diabetes. Endocrinology 2004;145: 2594-2603.

$11 \mathrm{Yu}$ C, Wang F, Kan M, et al. Elevated cholesterol metabolism and bile acid synthesis in mice lacking membrane tyrosine kinase receptor FGFR4. J Biol Chem 2000;275:15482-15489.

12 Shimada T, Kakitani M, Yamazaki Y, et al. Targeted ablation of Fgf23 demonstrates an essential physiological role of FGF23 in phosphate and vitamin D metabolism. J Clin Invest 2004;113:561-568.

13 Moore JW, Dionne $\mathrm{C}$, Jaye $\mathrm{M}$, et al. The mRNAs encoding acidic FGF, basic FGF and FGF receptor are coordinately downregulated during myogenic differentiation. Development 1991;111:741-748.

14 Kontaridis MI, Liu X, Zhang L, et al. Role of SHP-2 in fibroblast growth factor receptor-mediated suppression of myogenesis in C2C12 myoblasts. Mol Cell Biol 2002;22:3875-3891.

15 Marics I, Padilla F, Guillemot JF, et al. FGFR4 signaling is a necessary step in limb muscle differentiation. Development 2002;129:4559-4569.

16 Khan J, Wei JS, Ringner M, et al. Classification and diagnostic prediction of cancers using gene expression profiling and artificial neural networks. Nat Med 2001;7:673-679.

$17 \mathrm{Yu} \mathrm{S,} \mathrm{Zheng} \mathrm{L,} \mathrm{Asa} \mathrm{SL,} \mathrm{et} \mathrm{al.} \mathrm{Fibroblast} \mathrm{growth} \mathrm{factor}$ receptor 4 (FGFR4) mediates signaling to the prolactin but not the FGFR4 promoter. Am J Physiol Endocrinol Metab 2002;283:E490-E495.

18 Celli G, LaRochelle WJ, Mackem S, et al. Soluble dominant-negative receptor uncovers essential roles for fibroblast growth factors in multi-organ induction and patterning. EMBO J 1998;17:1642-1655.

19 Trinh DK, Zhang K, Hossain M, et al. Pax-6 activates endogenous proglucagon gene expression in the rodent gastrointestinal epithelium. Diabetes 2003;52: 425-433.

$20 \mathrm{Yu}$ S, Asa SL, Ezzat S. Fibroblast growth factor receptor 4 is a target for the zinc-finger transcription factor Ikaros in the pituitary. Mol Endocrinol 2002;16: 1069-1078.

21 Kostrzewa M, Muller U. Genomic structure and complete sequence of the human FGFR4 gene. Mamm Genome 1998;9:131-135.

22 Becker M, Brauninger A, Wolf G, et al. Identification and functional characterization of the human and murine fibroblast growth factor receptor 4 promoters. Biochem Biophys Res Commun 2000;276:493-501.

23 Ezzat S, Yu S, Asa SL. Ikaros isoforms in human pituitary tumors: distinct localization, histone acetylation, and activation of the $5^{\prime}$ fibroblast growth factor receptor-4 promoter. Am J Pathol 2003;163: 1177-1184.

24 Saito H, Kouhara H, Kasayama S, et al. Characterization of the promoter region of the murine fibroblast growth factor receptor 1 gene. Biochem Biophys Res Commun 1992;183:688-693.

25 Patel SG, Funk PE, DiMario JX. Regulation of avian fibroblast growth factor receptor 1 (FGFR-1) gene expression during skeletal muscle differentiation. Gene 1999;237:265-276.

26 Avivi A, Skorecki K, Yayon A, et al. Promoter region of the murine fibroblast growth factor receptor 2 (bek/ KGFR) gene. Oncogene 1992;7:1957-1962.

27 Parakati R, DiMario JX. Sp1- and Sp3-mediated transcriptional regulation of the fibroblast growth factor receptor 1 gene in chicken skeletal muscle cells. J Biol Chem 2002;277:9278-9285.

28 Pownall ME, Gustafsson MK, Emerson Jr CP. Myogenic regulatory factors and the specification of muscle progenitors in vertebrate embryos. Annu Rev Cell Dev Biol 2002;18:747-783.

29 Boilly B, Vercoutter-Edouart AS, Hondermarck H, et al. FGF signals for cell proliferation and migration through different pathways. Cytokine Growth Factor Rev 2000;11:295-302. 